\title{
La escuela normal y la voluntad científica de los nuevos educadores (Argentina, 1880-1900)
}

\author{
Alejandro Herrero* \\ herrero_alejandro@yahoo.com.ar
}

\begin{abstract}
Resumen
Los estudios de Juan Carlos Tedesco, Andrea Alliaud, Inés Dussel y Lucía Lionetti, para citar trabajos clásicos y más recientes, sobre la historia del normalismo en el siglo XIX, enfocan su mirada en las Memorias de Instrucción Pública, en publicaciones oficiales, y en publicaciones periódicas masivas. Ha escapado en la mayoría de los estudiosos el examen de las voces de los nuevos educadores y sus discusiones en las múltiples asociaciones creadas en las dos últimas décadas del siglo XIX. Uno de los aportes de mi investigación consiste en indagar las publicaciones de estas asociaciones. Analizo de qué manera estos nuevos educadores usan la ciencia para describir la realidad educativa y para legitimar, sobre todo, sus posiciones frente a los fundadores del normalismo. Se trata de una lucha al interior del campo educacional donde la voluntad científica de los nuevos educadores es invocada para autorizar su palabra y justificar el acceso a los mejores espacios de decisión.
\end{abstract}

Palabras clave: normalismo - educadores - política - Argentina.

\begin{abstract}
Juan Carlos Tedesco, Andrea Alliaud, Inés Dussel and Lucía Lionetti's stdies, just to mention but a few, about the history of normalis in the 19th century, focus their perspective on the Memoirs of Public Instruction and jounalist and official publications. The majority of schollars have left aside the analysis of the new educators' specches together with their discussions in the different associations created in the last two decades of the 19th century. My intention is to investigate the publications of such associations and to analyse how these new eduators use science to describe the educational reality and to legitimise their views before the founders of normalism. It is a struggle within the educational field where the scientific will of the new educators is cited to authorise their words and to justify the access to the best desicion arenas in the different fields of the educational system.
\end{abstract}

Keywords: schoolteacher- educator- politics - Argentina. Introducción

Diferentes investigadores señalan que en sede educativa, a nivel provincial y nacional, impera el poder de decisión de la dirigencia política gobernante, de los fundadores de las escuelas normales y de los colegios nacionales, y de educadores del movimiento educativo católico, ${ }^{1}$ sólo para citar los actores más relevantes. ${ }^{2}$

\footnotetext{
* Universidad Nacional de Lanús, Academia Nacional de Ciencia, CONICET.

${ }^{1}$ El movimiento educativo católico es fuerte en diferentes provincias, Santa Fe es un ejemplo emblemático y estudiado. El historiador Diego Mauro sostiene que la enseñanza religiosa en Santa Fe se mantiene también durante las primeras décadas del siglo XX, interrumpida en un breve lapso de tiempo: 1934-1935. Mauro, Diego, "Católicos, educación y política. La enseñanza religiosa entre la curia diocesana y las orientaciones educativas del estado provincial. Santa Fe, 1915-1937", en: Estudios Sociales, n. 36, Santa Fe, Universidad Nacional del Litoral, 2009.
} 
En las décadas de 1880 y 1890, nuevos educadores formados en las escuelas normales y colegios nacionales, luchan por imponer su palabra y por acceder a espacios de decisión en temas del magisterio y de la educación en general. Esto no significa que estén al margen de toda instancia de decisión y del uso de la palabra. He advertido en otras trabajos que, incorporados en el campo educativo, los nuevos educadores han fundado asociaciones nacionales y revistas de gran repercusión en el campo educacional, ${ }^{3}$ han creado escuelas particulares, han accedido a viajes al exterior para estudiar otros sistemas educativos financiados por el Estado, han propuesto nuevos temas en la agenda educativa y han impuesto, por ejemplo, la enseñanza del trabajo manual pedagógico en 1896 en el sistema educacional, ${ }^{4}$ se han incorporado en el equipo de trabajo de ministerios nacionales, por ejemplo durante el ministerio de Osvaldo Magnasco (1898-1901), ${ }^{5}$ y varios de ellos han ocupado el cargo de gobernador y cargos legislativos ${ }^{6}$ en sus provincias y en la nación. ${ }^{7}$ Los nuevos educadores tienen la palabra, y alcanzan cierto poder de decisión en un determinado nivel del campo educativo y del campo político, ocupando espacios tanto en la sociedad civil con sus asociaciones, publicaciones y escuelas particulares, como en algunos espacios del Estado nacional o Estados provinciales, pero aspiran (y lo dicen explícitamente), a los cargos más elevados en el Consejo Nacional de Educación, en la Dirección General de Escuelas en las provincias, y en el Ministerio de Instrucción Pública, entre otros. ${ }^{8}$

En esta lucha el saber es un capital decisivo: los nuevos educadores se presentan como los portadores de

${ }^{2}$ Alliaud Andrea, 1993; Barrancos Dora, 1990 y 1991; Biagini, Hugo, 1983; Bertoni, L., 2000; Cirigliano, Gustavo, 1967 y 1969; Chavarría, José Manuel, 1947; Colmenar Orzares, Carmen, 1983; Di Tella, Torcuato, 1965; Dussel, Inés, 1997; Herrero, Alejandro. 2010; Katzman, Rubén, 1968; Martínez Paz, Fernando, 1997; Lionetti Lucía, 2007; Montenegro Ana María, 2012; Morgade Graciela, 1997; Puiggrós, Adriana, 1991 y 2001; Ramos J. P., 1910; y Tedesco, Juan Carlos. 1982.

${ }^{3}$ Finocchio Silvia, 2009; y Herrero, Alejandro, 2011.

${ }^{4}$ Muzzopappa Héctor, 2012.

${ }^{5}$ Herrero, Alejandro, 2011.

${ }^{6}$ Leamos al menos uno de las tantas columnas que se repiten: "El número de Normalistas en el Congreso de la Nación aumenta rápidamente, y a este paso no estará lejano el día en que hubiese mayoría de maestros y profesores normalistas en las Cámaras Nacionales. Los que actualmente existen ocupando una banca del augusto recinto son: Pedro Barrasa en el Senado, por Santiago; en la cámara de diputados: Sergio Alvarado, por Jujuy, Gustavo Ferrari y Adolfo Castellanos, por Catamarca. En las últimas elecciones que acaban de efectuarse en toda la República han salido normalistas más como diputados: Lidoro Avellaneda por La Rioja, y M. Astrada por Córdoba. Ahora solo falta que esos profesores tan altamente colocados hagan oír su voz y hagan sentir su acción como dignos representantes de la cultura nacional". "Noticias. Normalistas en el Congreso", en: La Educación. Publicación Quincenal, Buenos Aires, Marzo 15 de 1896, año XI, n. 228.

${ }^{7}$ Herrero, Alejandro, 2010 y 2011.

8 "Permanente. En vista de que todos reconocen la facilidad de dar poderoso impulso a la enseñanza secundaria, llevando a los más afanados profesores a los puestos más importantes, y que estos solo puede hacerse cuando vaya al Ministerio de Instrucción Pública un verdadero educacionista, capaz de distinguir a los hombres idóneos con que cuenta el personal docente argentino, presentamos como candidatos para Ministro de Instrucción Pública a los doctores J. B. Zubiaur y J. A. Ferreira, quienes han dado pruebas inequívocas de capacidad, a juicio del país entero, que es el mejor juez cuando se trata de sus intereses más caros.

Habiéndose probado en Corrientes y en Entre Ríos que basta llevar a la Dirección General de Escuelas un Profesor de distinguido talento para que se realicen inmensos bienes, y como sería inexplicable que los gobiernos de Provincia se negasen a dar honra y gloria a su pueblo, cuando pueden hacerlo, nos permitimos señalar algunos profesores que podrían hacer obra imperecedera y que nos parece aceptarían el expresado cargo; son los señores Ernesto Bavio, Manuel Sarfield Escobar, Víctor Mercante, Angel C. Bassi, Juan Tufró, Marcelino Martínez, Maximio S. Victoria, Yole A. Zolezzi, José Bianco, Pedro Coracoche, Francisco Podestá, Porfirio Rodríguez." "Permanente", en: La Educación, periódico quincenal, 15 de febrero y 1 de marzo de 1896, año XI, n. 226-227. Esta sección, llamada permanente se reitera de manera constante en dicha publicación con este contenido o con otro, pero siempre indicando a los poderes públicos los nombres de la nueva escuela para ocupar puestos en el área educativa. Herrero Alejandro, 2010 
un saber científico 9 que exhiben como diferente y superador del saber de todos los actores que participan del campo educativo: superadora de los fundadores de las escuelas normales y de los colegios nacionales, de los educadores del movimiento educativo católico, de los educadores de las comunidades extranjeras, y de la dirigencia política, para indicar sólo algunos actores en pugna. El que está autorizado para hablar y para decidir debe acreditar, entre otras cuestiones, un saber que lo califique y legitime, un saber que le permita descubrir los problemas y pueda, sobre todo, resolverlos. La voluntad científica es uno de los rasgos que definen a los nuevos educadores, pero también es visible la voluntad democrática, la voluntad liberal, la voluntad patriótica y republicana, la invocación de un lenguaje religioso y de un lenguaje militar, entre otros, que forman parte del ideario del normalismo y no sólo de los nuevos educadores.

Mi hipótesis general, expuesta en otras investigaciones, ${ }^{10}$ supone que los diferentes registros argumentativos están asociados a luchas específicas, cada invocación de un saber (científico, democrático, liberal, patriótico, republicano, religión cívica etcétera) es utilizado para describir una realidad, justificar una determinada cuestión o interés y es interrumpido o violentado cuando lesiona un interés o principio caro al normalismo.

Siguiendo esta línea de investigación examino, de manera particular en este artículo, la voluntad científica de los nuevos educadores, los diversos usos de las ciencias que invocan en sus argumentos para legitimar su lugar en el campo educativo y para proponerse, al mismo tiempo, como continuadores y superadores de los fundadores del normalismo. Las fuentes seleccionadas (revistas de las asociaciones) casi no fueron consultadas por los estudiosos de la historia de los educadores normales ni del campo educativo; y son relevantes, tal como se intentará mostrar en este artículo, para mirar otros rasgos y otros conflictos del sistema de enseñanza de fines del siglo XIX argentino desde la perspectivas e intereses de asociaciones educativas civiles y no desde el Estado.

El escrito se divide en dos partes: primero exploro en las revistas de sus asociaciones (más particularmente de la Asociación Nacional de Educación, en adelante: ANE), de qué manera los nuevos educadores usan la ciencia (o mejor: argumentos de ciertas autoridades científicas) para proponerse como profesionales mejor preparados que los fundadores de las escuelas normales, y en segundo lugar, indago las discusiones al interior de algunas asociaciones de los nuevos educadores.

\section{Los fundadores de las escuelas normales y la voluntad científica de los nuevos educadores}

El maestro, para los nuevos educadores, es un científico y un artista, alguien que posee las destrezas y los conocimientos específicos de la ciencia y del arte de la enseñanza. ${ }^{11}$ Subrayan, siguiendo estas premisas, que las escuelas normales son las únicas sedes educativas que están preparadas para la formación de maestros y profesores. Los nuevos educadores invocan una pedagogía científica que los lleva a postular que cada

\footnotetext{
${ }^{9}$ Oscar Terán ha escrito varios libros sobre la historia de las ideas políticas y culturales en Argentina y en Latinoamérica usando la expresión positivismo. Sin embargo, en su última contribución ha decidido utilizar la expresión cientificismo puesto que es la ciencia, en todas sus acepciones, el vocablo que se utiliza generalmente, con la excepción por ejemplo de la revista "Escuela Positiva" de la provincia de Corrientes. Terán Oscar, 2000. Sobre el positivismo y más particularmente sobre el positivismo pedagógico pueden consultarse estos estudios clásicos y recientes: Filippa, Nely, 2004; Perelstein, Berta, Tedesco, Juan Carlos. 1973; 1973b y 1985; 1952; Terán Oscar, 1986; 1987; 2000 y 2008 ; y Soler, R, 1959.

${ }^{10}$ Herrero, Alejandro, 2010 y 2011.

11 "Lo que debe ser el maestro. Necesidad de las escuelas normales por el Profesor Normal D. Ernesto Bavio", en: La Educación. Periódico Quincenal, Buenos Aires, marzo 1, 1886, año 1, n. 1; La Educación. Periódico Quincenal, Buenos Aires, marzo 31, 1886, año 1, n. 3; y "La ciencia y el arte de la educación", en: Revista Pedagógica Argentina. Órgano del Centro Unión Normalista, Buenos Aires, año III, junio 1890, n. XXII.
} 
alumno accede a la verdad personalmente, y el maestro debe guiarlo a esa meta, que se accede a partir de una experiencia, y mediante una demostración empírica. Hablan de la libertad y de la actividad del nińo para descubrir la verdad, en oposición a la postura de los fundadores del normalismo, a sus ojos, erudita, repetitiva, que ve la cabeza del nińo como un recipiente que hay que llenar con conocimientos, y forma, invariablemente, un individuo pasivo. La vieja escuela, en la opinión de los nuevos educadores, instruye, apela a los libros, a la repetición y la memorización, en lugar de educar y de instruir. ${ }^{12}$ Si la voluntad normalista (defensa de los valores e intereses de la escuela normal) une a fundadores y nuevos educadores, la concepción pedagógica asociada a un tipo particular de voluntad científica y al arte de enseńar invocada por los nuevos educadores los separa.

Por razones de espacio me detendré en la voluntad científica de los nuevos educadores (tema central de este artículo), asociada por lo general con la fisiología (disciplina de enorme prestigio a fines del siglo XIX). ${ }^{13}$ Para ilustrar esto indicaré algunos ejemplos emblemáticos.

En la agenda política y social ${ }^{14}$ de fines de siglo se discute si los nińos deben trabajar en los talleres o concurrir a las escuelas. ${ }^{15} \mathrm{La}$ argumentación de los nuevos educadores se apoya en autoridades científicas (fisiólogos principalmente). Invocan una multiplicidad de datos y estadísticas de estudios científicos sobre el desarrollo de los músculos de nińos y de personas adultas, y concluyen que un niño no puede trabajar en un taller porque sus músculos aún no están formados, y que su lugar es la escuela. No habla el nuevo educador, sino la ciencia, o mejor los estudios de los fisiólogos, la experiencia empírica de los investigadores en sus consultorios ofrecen esos resultados invocados por los nuevos educadores. Algo parecido sucede cuando se plantea la elección de los bancos en las escuelas y su relación con la salud física de los niños: se postula que sólo docentes y médicos están preparados para hablar y decidir sobre esta cuestión. ${ }^{16}$ También aquí la voz de los fisiólogos y de los educadores se complementan: son dos caras de la misma moneda.

Sin embargo, al introducir el tema de la niñez y la moral, los nuevos educadores se separan de Spencer, autoridad siempre citada positivamente, quién sostiene que la moral está ligada a la herencia biológica. ${ }^{17}$

12 González Emilio, “Menos libros y más observación”, en: Revista Pedagógica Argentina, Órgano del Centro Unión Normalista, Año III, Buenos Aires, Julio de 1890, n. XXIII, p. 403-405; Ferreira J. A., "Educación Pública", en: Revista Pedagógica Argentina, Órgano del Centro Unión de Normalistas, Año III, Buenos Aires, Mayo 15 de 1891, n. 28, p. 6-9; "Prólogo de una obra inédita de los profesores normales Andrés Ferreira y Luis Suarez. Educación del Ciudadano", en: Revista Pedagógica Argentina, Órgano del Centro Unión de Normalistas, Año III, Buenos Aires, Mayo 31 de 1891, n. 29, p. 19-22; "Textos de enseñanza primaria", en: Revista Pedagógica Argentina, Órgano del Centro Unión de Normalistas, Año III, Buenos Aires, Agosto 15 de 1891, n. 34, pp. 97-101; "Sobre el mismo tema. Textos de enseñanza primaria", en: Revista Pedagógica Argentina, Órgano del Centro Unión de Normalistas, Año IV, Buenos Aires, Agosto 31 de 1891, n. 35, pp. 113-115; "Sobre el mismo tema. Textos de enseñanza primaria", en: Revista Pedagógica Argentina, Órgano del Centro Unión de Normalistas, Año IV, Buenos Aires, Septiembre 15 de 1891, n. 36, pp. 128-131; “Sobre el mismo tema. Textos de enseñanza primaria”, en: Revista Pedagógica Argentina, Órgano del Centro Unión de Normalistas, Año IV, Buenos Aires, Septiembre 30 de 1891, n. 37, pp. 144-147; "Sobre el mismo tema. Textos de enseñanza primaria", en: Revista Pedagógica Argentina, Órgano del Centro Unión de Normalistas, Año IV, Buenos Aires, Octubre 15 de 1891, n. 38, pp. 161-163; "Lecciones prácticas", en: Revista Pedagógica Argentina, Órgano del Centro Unión de Normalistas, Año IV, Buenos Aires, Octubre 30 de 1891, n. 39, p. $184-185$.

${ }^{13}$ Biagini Hugo, 1985; Terán Oscar, 1987; y Zimmermann Eduardo, 1995.

${ }^{14}$ Biagini Hugo, 1985; Recalde Héctor, 1997; y Terán Oscar, 1987; y Zimmermann Eduardo, 1995.

${ }^{15}$ En 1899, dos nuevos educadores miembros del Consejo Nacional de Educación, B. Zubiaur y J. V. González, escriben un informe muy negativo sobre la deserción escolar, señalada como la gran dificultad de la educación primaria, y destacan con enorme alarma, además, en las lamentables condiciones que trabajan niños y mujeres en diferentes empresas. Censo escolar del Distrito IV. Noviembre de 1899. Publicación del Consejo Nacional de Educación por el Dr. Joaquín V. González, vocal del Consejo, (1900). Félix Lajouane Editor, Buenos Aires.

16 "Bancos escolares", en: Revista Pedagógica Argentina, Órgano del Centro Unión de Normalistas, Año IV, Buenos Aires, Septiembre 30 de 1891, n. 37, pp. 153-154.

17 "El desarrollo del sentimiento moral en el niño", en: Revista Pedagógica Argentina. Órgano del Centro Unión de Normalistas, año III, n. XXV, octubre 1890, pp. 520-522; y año III, n. XXVII, diciembre de 1890, pp. 589-592. 
Aceptar esta premisa spenceriana significa que la educación no tiene sentido, no cumple ninguna función, puesto que todo está dado por la naturaleza. A una argumentación científica responden con otra del mismo registro: invocan a Darwin y otras autoridades para atacar la posición spenceriana. Se critica a un científico desde una argumentación científica, y siempre los intereses y los valores del normalismo imperan en sus discusiones. Voluntad normalista y voluntad científica se articulan en sus discursos.

En el artículo "La tartamudez y el maestro" de J. Katzenstein se condensan varios temas mencionados y se subraya una nueva cuestión: la escuela sana. ${ }^{18}$ Un eje se retira: la defensa de la escuela normal como la mejor y única sede para la formación de maestros y profesores, y la presentación de los nuevos educadores como los portadores de un saber más adecuado que la de los fundadores del normalismo. ${ }^{19}$ Sus destinatarios son los maestros y profesores egresados de escuelas normales, a quienes les señala que están mal formados porque no saben qué es la tartamudez. No saben (porque no se les ha enseńado) que la escuela es un lugar de educación y de sanación.

Si habría un organismo defectuoso, habría balbuceo, y la cura se la da el médico, explica Katzenstein, pero la tartamudez no es por un organismo defectuoso, sino su "empleo impropio", por lo tanto la cura la da el que enseńa como emplear apropiadamente los 'órganos", la cura de la tartamudez del niño es propia del maestro. Si los fisiólogos ${ }^{20}$ llevan problemas particulares de sede educativa al consultorio, en este caso se advierte el proceso inverso: los maestros llevan un tema médico a sede escolar, en este caso la tartamudez, porque sólo el maestro puede lograr la sanación del niño. Katzenstein realiza, en el campo científico, un desplazamiento, la tartamudez no es un problema solamente médico ni la respuesta proviene del consultorio ni de un tratamiento ni requiere medicamentos, sino que, en determinados casos, es un problema educativo, se detecta y se cura en las aulas y el maestro cumple una función fundamental en la cura de esta enfermedad. ${ }^{21}$ Invoca los estudios de los fisiólogos para explicar qué es la tartamudez, y al mismo tiempo, invoca las experiencias en sus consultorios para evidenciar que no pueden resolver el problema de esta enfermedad en los nińos. Les da la palabra a los fisiólogos para que el lector educador pueda escuchar que los mismos médicos admiten que visualizan el problema pero que no pueden sanar. ${ }^{22}$ Finalmente Katzenstein

\footnotetext{
${ }^{18}$ Katzenstein Jorge, "La tartamudez y el maestro", en: La Educación, periódico quincenal, Buenos Aires, agosto de 1887. Año II, n. 34, pp. 541-543.

${ }^{19}$ Katzenstein Jorge, Investigaciones. Sobre nuestro sistema de formar maestros y la necesidad de cambiarlo, pp, 120931295. La educación, 15 junio 1889. Continúa: pp. 1322-1235. La Educación, 15 de julio 1889.

${ }^{20}$ En las publicaciones de los nuevos educadores se reproducen estudios de fisiólogos que discuten si las mujeres pueden estudiar en las universidades: "La instrucción superior de la mujer. Opinión de los médicos", en: La Educación, periódico quincenal, Buenos Aires, enero-febrero de 1887. Año II, n. 22-23, pp. 357-358. Este es uno de los tantos temas educativos que se ocupan los fisiólogos, sólo he indicado un ejemplo que exploro en la segunda parte de este trabajo, y cito uno de los muchos estudios que se reproducen en las revistas de los nuevos educadores.

21 “¿Quién debe combatir la tartamudez: el médico o el maestro? Si solo veinte años atrás se hubiera dirigido esta pregunta a cualquiera de los maestros de este país, seguramente su contestación hubiera sido, el médico. Hoy esto es distinto. En diferentes ocasiones he traído la conversación con maestros a este terreno y he notado con satisfacción que casi siempre comprendían la importancia del maestro para combatir la tartamudez de los niños. Esta convicción es sin duda el resultado de la preparación que desde cierto tiempo reciben en las escuelas normales los que se dedican a la enseñanza [...] Pero he notado también que esos mismos maestros no tenían ninguna claridad de ideas respecto a las verdaderas causas del mal en cuestión, y que por consiguiente, tampoco podían explicarse respecto a los medios que pondrían en juego si se les ofreciese el caso de tener que poner en práctica su curación”. Ob. Cit., pp. 541-542.

22 [...] son cuatro los factores que intervienen en el habla: el cerebro y los nervios, los pulmones, la voz, y los órganos de articulación. Supongamos ahora que la tartamudez fuese causada por la desordenada actividad del cerebro, y veremos que podría este factor intervenir de dos modos: por un lado podrían los pensamiento seguirse con tal precipitación que su reproducción audible se haría imposible, por otro lado podrían tomar forma sin energía y extremadamente pesada y su transmisión por los nervios a los otros factores se haría del mismo modo, sin energía y con pesadez. ¿Qué puede en estos casos hacer el maestro? En el primer caso debe rodear el habla, y por lo tanto también los pensamientos del niño, con un círculo rígido de reglas que le obligue a pensar y hablar ordenadamente y con tranquilidad. En el segundo caso
} 
sintetiza lo que quiere afirmar: la respuesta al problema de la tartamudez en los nińos no proviene en la mayoría de los casos, según la experiencia científica, del mundo médico sino del mundo escolar, y los fundadores de las escuelas normales lo ignoraban.

Ahora bien, la voluntad científica y estos usos de la ciencia o de estudios científicos no sólo se visualizan en la lucha de los nuevos educadores contra los fundadores de las escuelas normales o contra científicos que atacan intereses y valores caros al normalismo, también se pueden apreciar en sus propias discusiones.

\section{Discusiones en la Asociación Nacional de Educación}

Sus diferencias y enfrentamientos comienzan en los primeros números de La Educación, órgano de difusión de la ANE, creada por nuevos educadores en 1886. Me detendré, a modo de ejemplo, en una de sus polémicas. ${ }^{23}$

No tienen firma los artículos que defienden la desigualdad de los sueldos entre hombres y mujeres, sin embargo se puede inferir que se trata del mismo director de la revista La Educación. Pero más allá si se trata del director está claro que es una opinión de miembros de la publicación y de la ANE. La respuesta emerge de la Asociación Progresista (en adelante: AP), ${ }^{24}$ una asociación creada por docentes mujeres de la escuela normal de Mendoza, que adhiere a las bases de la ANE en cuanto a la defensa de las escuelas normales y de los intereses y valores caros al normalismo, pero que sale a dar batalla al interior de la propia ANE avalando la igualdad de los sueldos, entre otras cuestiones.

debe incitar a mayor actividad intelectual, al vacilante; debe ayudarle a pensar con más energía, y así transmitir actividad y energía a los otros factores del habla. Considerando ahora al segundo factor, los pulmones, encontramos que también interviene de un doble modo en la tartamudez. Una vez los pulmones impelen el aire necesario para el habla demasiado de golpe, otra lo retienen al principio o en medio de la frase o de la palabra; y estos defectos de la respiración al hablar son mayormente debidos al diafragma que es el regulador de esta función. Para combatir esta defectuosa respiración los médicos han empleado aparatos neumáticos de diferentes especies, pero sin resultados satisfactorio. Lo que más conviene son determinados ejercicios gimnásticos que aumentan la fuerza del diafragma de los pulmones y de la caja torácica, y que transforme su actividad mecánica en actividad conciente y arreglada. Es preciso llamar la atención del niño sobre su defectuosa respiración cuando habla, y obligarle, mediante un control minucioso, a efectuar este acto de un modo natural. Todo esto corresponde al maestro y no a los médicos. Veamos ahora si se necesita del médico tratándose del tercer factor, el aparato vocal. [...] Nada han recomendado los médicos respecto al desordenado movimiento de las cuerdas vocales, cuya influencia en la tartamudez no hace mucho que fue reconocida. Lo que debe hacer el maestro, es inducir al niño a coordinar el golpe de la voz con la expulsión del aire, a cuyo efecto debe también hacerle practicar una especie de gimnástica vocal. Este defecto es más difícil de corregirlo que los otros dos que influyen en la tartamudez, y debe dedicarse a él mayor cuidado. (p. 542) Quédanos el cuarto factor que son los órganos de articulación. [...] El empleo impropio de los órganos de articulación es el más fácil de corregir. El maestro debe hacer hablar al niño con la boca bien abierta, y cuando ha conseguido esto, habrá desaparecido también el defecto de cerrarse el canal a destiempo. Después solo le resta enseñar al niño la correcta pronunciación de cada letra. Empero queda aún un enemigo poderoso que no puede dejarse de toma en consideración [...] Este peligroso enemigo del buen habla son las afecciones mentales, sobre todo la vergüenza, el miedo y el terror. La inutilidad de medicamentos para combatir este enemigo es óbvia; solo la educación puede conseguir amortiguar semejantes impresiones; solo ella puede fortalecer la confianza del niño, en el mecanismo del habla de tal manera, que el tartamudeo, aún en situaciones críticas, quede dueño de su habla. De todo lo expuesto resulta, que la tartamudez debe ser combatida por el maestro mediante una adecuada instrucción y educación; y sería de desear que en las escuelas normales se dedicara alguna parte del tiempo, para iniciar a los aspirantes al magisterio en los procedimientos y métodos más recomendados que se aplican en esta parte especial de la enseñanza". Ob. Cit., p. 543.

${ }^{23}$ Sobre las publicaciones de educación en la historia argentina puede consultar el libro de Finocchio, Silvia, 2009.

24 "Asociación de Educación en Mendoza”, en: La Educación, periódico quincenal, Buenos Aires, julio de 1886. Año I, n. 11, p. 169; y “Correspondencia de Mendoza”, en: La Educación, periódico quincenal, Buenos Aires, setiembre de 1886. Año I, n. 14 y 15, p.232. 


\section{Voluntad científica, salarios de los docentes y cargos directivos}

El primer artículo se edita en el primer número de La Educación y da pie a la polémica. ¿Cuál fue la piedra del escándalo? El o la articulista informa que en 1885 el Congreso votó "una partida con el objeto de igualar los sueldos de las maestras y los maestros", y se confiaba "a maestras la dirección de escuelas elementales y superiores de varones." 25

El o la docente que escribe tiene una mirada negativa y alarmada al sostener que estas disposiciones están "contra nuestros hábitos, son perjudiciales a los intereses generales de la sociedad, aunque maestras y maestros lo acepten con satisfacción." ${ }^{\prime 26}$ ¿Que se está discutiendo? La igualdad entre maestras y maestros y el acceso de docentes mujeres a cargos de dirección en escuelas de varones. La discusión se produce en el lugar más decisivo, el Congreso: desde 1885 se propone la igualdad de los sueldos como una política del Estado.

¿Qué estrategia adopta el o la articulista? Primero deja ver su aparente debilidad argumentativa, la dirigencia política toma esta iniciativa y es apoyada con entusiasmo por maestros y maestras, para después señalar el abismo que se abre con semejante medida que atenta, afirma, contra los hábitos e intereses de la propia sociedad. Ve y hace ver no sólo que se parte de una premisa falsa, sino que el problema es mayor puesto que los educadores la reciben con gran entusiasmo sin percibir que es un peligro social.

Desde los escritos de 1840 de Domingo Faustino Sarmiento, (primer capítulo de Educación Popular, o la parte dedicada a los Estados Unidos de América de sus Viajes) se puede apreciar este eje (la relación educación y sociedad moderna, educación y buenos gobiernos) que sostiene y legitima el discurso de los educadores. Sólo hombres y mujeres que sepan leer y razonar pueden conocer y ejercer sus derechos como habitantes y ciudadanos de una sociedad y un estado moderno. El articulista acude a este eje del discurso educativo del siglo XIX que nadie discute sino que por el contrario define y fundamenta todo razonamiento de un educador y de un normalista: los desórdenes sociales y los malos gobiernos tienen su origen en las malas escuelas y tienen su respuesta en las buenas escuelas. ¿Cómo usa el o la articulista este eje básico del discurso educativo y normalista? No avanza su argumentación por el lado educativo, sino que su razonamiento se inicia y se desarrolla por el lado social. Primer desplazamiento: es una medida que apunta a las maestras, a su salario, al acceso a cargos directivos, sin embargo, no es leída en una primera instancia en clave educativa (como un logro para los educadores), sino en clave social, y en un tono alarmista puesto que lesiona, a sus ojos, lo más fundamental: la sociedad misma. Segundo desplazamiento: de la clave social (luego dirá sociológica) se desplaza a la clave fisiológica para sostener, una vez más, la desigualdad entre hombres y mujeres. Afirma que "la mujer no puede poseer, por razón de su constitución misma, un conjunto de facultades mentales tan poderosas como el hombre, y ser susceptible, por consiguiente, de igual desarrollo y perfección. ${ }^{27}$ Esta desigualdad que ubica al hombre por encima de la mujer, luego se invierte cuando alude a los grados inferiores de la enseñanza, en este caso señala que "la mujer es superior para el magisterio en la instrucción de la infancia, por las condiciones mismas de su naturaleza." ${ }^{28}$ Se subraya que la naturaleza ha establecido esta desigualdad: la desigualdad, de este modo, no es producto de la mirada del o de la articulista sino de la naturaleza, por lo tanto no habla el que habla sino que habla la naturaleza y las investigaciones de los fisiólogos. Su objetivo es sostener el siguiente argumento: no se puede discutir al que habla sino que hay que discutir con la naturaleza y con la ciencia (investigaciones de los fisiólogos).

\footnotetext{
25 “Maestras y maestros”, en: La Educación, periódico quincenal, Buenos Aires, marzo 1 de 1886. Año I, n. 1, pp. 6-7.

${ }^{26}$ Ibidem.

${ }^{27}$ Ibidem.

28 “Ha formado la naturaleza a la mujer, sensible y afectuosa, y el niño, en los primeros años, necesita principalmente de estos estímulos, por lo mismo que sus facultades están aún en desarrollo y no puede discernir ni comprender otros. La observación de la infancia limitase a las cosas comunes, y si estas han de servir de base a la educación, ningún maestro puede reemplazar a la mujer en esta obra" Ibidem.
} 
Expuesto este discurso (son las voces de la naturaleza y de la ciencia las que hablan), el o la articulista desprende otra afirmación: si en los primeros grados los nińos necesitan las cualidades que sólo ofrecen las madres, en los grados superiores los nińos necesitan de las cualidades que sólo pueden ofrecer los padres. Sostiene, por ejemplo, que cuando una familia está a cargo sólo de la madre prima el desorden familiar "porque falta allí el padre que aquella no puede reemplazar. Robustece su argumento al asociar a la familia con una imagen escolar, una familia es "una escuela en pequeño", en ella deben aunarse estas dos fuerzas para dirigir convenientemente la instrucción, pero cada una en su esfera natural." ${ }^{29}$ Maestras y maestros, madres y padres, tienen funciones fundamentales, pero cada uno ocupando su lugar, cumpliendo su papel. ${ }^{30}$

El eje articulador es la relación educación y sociedad moderna, por lo tanto la clave fisiológica es invocada pero subordinada a la clave social y a la clave educativa; y siempre es la clave educativa la que domina en el discurso. Por este motivo, el o la articulista hace este nuevo giro en su argumentación. Primero la clave fisiológica antes invocada se lee en clave social: seńala que los maestros egresados de escuelas normales optan por otras profesiones porque el mal salario docente. Esto implica, en segundo lugar, que las escuelas son malas porque les falta la pieza fundamental que las sostiene, los maestros. ${ }^{31}$ De este modo se explica, finalmente, los problemas sociales y políticos, puesto que la desobediencia, la indisciplina en la sociedad tiene su origen en "las malas escuelas". ¿Qué quiere subrayar el o la articulista? Un punto fundamental de los educadores: que el origen y la solución de los problemas sociales y políticos están en las escuelas. ${ }^{33}$ Sólo ofreciendo un buen salario a los maestros egresados de las escuelas normales se puede dar una respuesta para que los maestros opten por el magisterio y las escuelas cumplan su función social y política.

Impera la voluntad normalista y la lucha al interior del normalismo. La clave sociológica o la clave fisiológica pueden leer una parte del problema, no pueden ver la totalidad tal como la hacen ver los educadores; sólo ellos están autorizados para hablar y para decidir porque son los que pueden ver y hacer ver, y los que resuelvan los problemas de la sociedad y de los gobiernos. Pero no todos los educadores pueden ver, porque él, o la articulista, sostienen que maestros y maestras celebran la igualdad de los sueldos que implica la disolución social. Hay, al mismo tiempo, una lucha en el campo educativo entre los que pueden ver y los que no pueden ver. Estos últimos están equivocados, a los ojos del o la articulista, y son educadores que producen un gran dańo a la sociedad y al campo educativo porque se trata de educadores que no cumplen su función social y política.

\section{Respuesta de la Asociación Progresista de Mendoza}

\footnotetext{
${ }^{29}$ Ibidem.

${ }^{30} \mathrm{Y}$ luego sostiene que "[...] en estos grados de la instrucción hay ciertas materias que no pueden, por lo menos actualmente que no poseemos un número crecido de maestras instruidas, aunque las haya muy anhelosas en sus deberes profesionales, enseñar como el hombre, tales como las ciencias naturales y matemáticas." Ibidem.

31 "En cuanto a la carencia de maestros, que motivan las disposiciones ya citadas, y a la influencia legítima que este debe tener en la obra de la educación, citaremos aquí la opinión de otro respetable maestro americano, M. Store, superintendente de Worcester, Massachussets: "No hay más cierto que el triste decrecimiento de la eficacia de las escuelas públicas, causada por el hecho de alejar de la profesión a tantos hombres. A la cabeza de toda escuela grande se necesita un hombre da vastas miras, juicioso y culto; su influencia es tan esencial a la conveniente formación del carácter en la escuela, como la influencia del padre en la educación de la familia [...]" Ibidem.

32 Ibidem.

33 "Y este mal es muy antiguo, cuya influencia en las clases educadas dirigentes de nuestra sociedad, ha trascendido y hoy aun actúa en nuestra sociabilidad. El respeto, la obediencia en los que gobiernan y en los que están subordinados a las prescripciones las más elementales de una sociedad regularmente construida, son la excepción. Su falta es la clave, la explicación más tangible de este fenómeno sociológico: una sociedad que vive cincuenta años en perpetua guerra interna para adquirir su libertad, si conseguirla aun, y que a cada momento nos presenta signos de volver a su pasado. Son las malas escuelas, las escuelas sin disciplina, que obran de esa manera." Ibidem.
} 
La respuesta provine de una educadora ${ }^{34}$ de Mendoza: apunta, en primer lugar, al estudio de los fisiólogos: La experiencia nos ha demostrado que las facultades intelectuales de la mujer no son inferiores a las del hombre, como lo aseguran algunos fisiólogos; pues, si bien es verdad que tiene, generalmente, menos desarrollado el cerebro de la mujer en su parte anterior, sabemos que la energía de las funciones no depende exclusivamente del tamańo de los órganos, sino también de su irritabilidad, y estando dotada la mujer de una irritabilidad más pronta, suple ésta a la falta de desarrollo de aquel. ${ }^{35}$

Si los fisiólogos invaden el espacio educativo, la educacionista invade el espacio de los fisiólogos, ataca sus razonamientos y estudios empíricos, utiliza sus categorías (la educacionista también sabe de fisiología porque es científica, sabe pensar científicamente), y arriba a conclusiones diametralmente opuestas. La educadora discute en el terreno de la fisiología de igual a igual con los fisiólogos, y no sólo cuestiona sus estudios, sino que les enseña a leer sus propias investigaciones:

Esto mismo se nota en muchos hombres más inteligentes que otros cuyo cerebro es mucho mayor. Además, no hay que apreciar la masa en su volumen absoluto, sino el relativo; luego, siendo el cuerpo de la mujer menor que el del hombre, tiene que serlo también en su masa cerebral $[. .$.$] Vemos que una persona organizada de la manera más perfecta, permaneciendo en$ la inacción, mengua la actividad de sus órganos: ahora bien, puede suceder que no cultivando la mujer ciertas facultades, los órganos correspondiente mengüen por falta de ejercicio, siendo por consiguiente, un efecto lo que se considera como causa. Se deduce de aquí que la inferioridad de la mujer depende de la falta de gimnasia intelectual indispensable para aprender a combinar ideas y de ninguna manera por defectos orgánicos; luego, educando a la mujer, llegará hasta donde el hombre llega, y prohibiéndole que cultive su entendimiento, se convertirá en un ser de perturbación la que debía serlo de armonía y bienestar. ${ }^{36}$

Luego de señalar que confunden la causa con el efecto, la educadora expresa claramente lo que quiere afirmar: "La mujer puede, pues, ejercer toda profesión que no exija mucha fuerza física y que no perjudique la ternura de su corazón." ${ }^{37}$ La educadora se desplaza, finalmente, del terreno de la fisiología al terreno del aula; para sostener que las claves científicas de la fisiología y de la educación arriban a la misma verdad: la "supuesta" superioridad de los varones no se ve en el aula, y la superioridad o inferioridad de una persona lo establece su nivel educativo, por lo tanto, la discusión no debe llevarse a cabo en el terreno de la fisiología sino en el terreno de la educación. ${ }^{38}$ Vale decir, en cuestiones de enseńanza y de escuelas la palabra la tiene el educador o la educadora y no los fisiólogos, la voluntad normalista impera siempre en el discurso de los nuevos educadores y de las nuevas educadoras.

\footnotetext{
34 Billorou, María José, 1997; Colmenar Orzares, Carmen, 1983; Lionetti Lucía, 2007; Morgade Graciela, 1997; y Szir Sandra. (2007). Desde un punto de vista más general sobre la historia de la mujer en Argentina son muy útiles los estudios de Barrancos, Dora, 1990; 1991; y 1993. La bibliografía sobre la historia de la mujer en Argentina es abundante, sólo cito a una de sus principales historiadoras.

35 "Diferentes profesiones que puede tener la mujer en Mendoza", en: La Educación, periódico quincenal, Buenos Aires, setiembre de 1886. Año I, n. 14 y 15, pp.232. y 235-237.

${ }^{36}$ Ibidem.

${ }^{37}$ Ibidem.

38 "Si fuera necesario únicamente igualdad de volumen para que la energía de las funciones fuesen las mismas, la inferioridad de la mujer se vería en todo. Sus sentidos serían torpes, menor su disposición para las artes, ciencias, etc., pero nada de esto sucede, la diferencia intelectual empieza con la educación. Los maestros de primeras letras jamás han notado superioridad en las facultades intelectuales de los varones; ni entre la gente ruda del pueblo en quienes la ecuación esta en ambos sexos igualmente descuidada, se ha notada la supuesta superioridad." Ibidem.
} 
Otra docente de la AP edita un escrito, estrechamente vinculada con esta conferencia: plantea la creación de una Escuela de Artes y Oficios para mujeres en Mendoza con el objetivo de lograr la emancipación social femenina. Su intervención apunta a destruir varias normas de percepción en el campo educativo y en el campo normalista más específicamente. Primero cuestiona la concepción educativa de los fundadores de las escuelas normales (basta recordar que Sarmiento se opone a la creación de escuelas de artes y oficios durante su gestión en 1880 como Superintendente de Escuelas). ${ }^{39}$ Segundo, apunta que la desigualdad entre el hombre y la mujer reside en que la mujer no se la prepara para el trabajo, y sólo el trabajo permite que la mujer se independice. Tercero, pone el acento en una cuestión que se opone a los fundadores del normalismo (Sarmiento plantea que la mujer representa menos costo en sueldos que el hombre) el magisterio sólo le da el saber a la mujer pero no le da el poder asociado al dinero que se gana en el mundo del trabajo, único elemento que permite la emancipación social. Y cuarto, sólo una mujer que se sostiene con su trabajo (del cuál obtiene un salario que la emancipe) puede, a su vez, sostener al Estado y sostener a su familia. ${ }^{40}$ Esta propuesta tenía tres destinatarios: los fisiólogos que se oponen a que la mujer estudie en las universidad o se desempeñe en cualquier profesión, los educacionistas de la ANE que comparten estos presupuestos y se apoyan en los estudios de estos fisiólogos, y los fundadores del normalismo que de la mano de Sarmiento rechazaban la creación de las Escuelas de Artes y Oficios. ${ }^{41}$

Siguiendo esta línea, en junio de 1889 se celebra en la ANE un hecho inédito: una educadora egresada de la escuela normal de profesoras de la ciudad de Buenos Aires se recibe de doctora en medicina, y se convierte en la primer doctorada argentina. ${ }^{42} \mathrm{El}$ texto celebratorio habla de la primera doctorada del país pero todo el tiempo se recuerda que es egresada de una escuela normal, que ha sido formada en la práctica de la observación y experimentación, y que es argentina: motivos que articulan el discurso de las asociaciones de los jóvenes educadores. ${ }^{43}$

${ }^{39}$ Sarmiento, D. F., 1881 y 1885.

40 "Nuestros educacionistas han dado hasta hoy en la educación femenina una preferencia marcada a los principios morales y al saber abstracto, olvidándose de su aplicación práctica: nada hay en ella que estimule a un trabajo que pueda independizar, porque no todas pueden dedicarse al magisterio: ella solo da el saber, pero jamás el poder: la educanda solo recibe una preparación teórica que la deja desamparada ante el trabajo que tantas veces le exige la imperiosa lucha por la existencia propia. La mujer entre nosotros no tiene más medio de sostén que su trabajo manual, y aún solo es fruto de un aprendizaje empírico [...] la primera e ineludible exigencia que hace la sociedad a cada uno de sus individuos para garantir su existencia y desarrollo, es, que aporte al tesoro común un capital o una producción de valor suficiente para llenar los deberes indispensables de sostén de su propia existencia en la vida de que goza [...] la mayoría del sexo femenino entre nosotros es incapaz de presentar este capital efectivo o en renta como resultado de su trabajo [...] el mayor y más amplio desarrollo de las facultades del hombre en el sentido productor de su trabajo, le da esta superioridad natural, que la mujer misma le concede; pues esta superioridad en el hombre nace, además, del abandono en que hasta hoy se ha tenido la educación de la mujer en relación a su posibilidad de producir [...] Recuperar para la mujer el rango que como madre le corresponde; independizarla de la humilde condición a que hoy se ve reducida por su inutilidad en el desarrollo social y garantirle la tranquilidad en la vida, es el ideal supremo a que aspira la institución de una Escuela de Artes y Oficios." Salomé Duffour, "Escuelas de artes y oficios para niñas y mujeres", en: La Educación, periódico quincenal, Buenos Aires, noviembre de 1886. Año I, n. 18, pp.284-287. La discusión continúa, sólo mencionaré dos artículos más. Una idea negativa: "La mujer madre y la mujer doctora", en: La Educación, periódico quincenal, Buenos Aires, noviembre de 1886. Año I, n. 18, pp. 290-292. Y una respuesta a este artículo por Felipe Caronti Casati, "La educación de la mujer" en: La Educación, periódico quincenal, Buenos Aires, diciembre de 1886. Año I, n. 20 y 21, pp. 322-324.

${ }^{41}$ Sarmiento, D. F., 1881 y 1885.

${ }^{42}$ La Educación. Periódico Quincenal, junio 1 de 1889. Año IV, n. 77, p. 1286.

43 "Cabe a una hija de la provincia de Entre Ríos, la Señorita Cecilia Grierson, el alto honor de ser la primer mujer argentina que, sobreponiéndose a las preocupaciones sociales, dedica su actividad y su labor paciente a los estudios científicos que abrazan las profesiones liberales. Dedicada durante seis años consecutivos -después de haber hecho su aprendizaje preparatorio en la Escuela Normal de Profesoras de esta Capital -al estudio de las ciencias médicas, ve 
El o la articulista da la palabra a la doctora, quién señala que encontró menos dificultades que las que esperaba y elogia a sus compañeros y profesores. ${ }^{44}$ Es la voz de la misma experiencia, es la voz de la observación y experimentación, es la voz de la que fue testigo de todo el recorrido del cursado y finalización de la carrera de medicina y lo puede contar. Este hecho discursivo habilita la palabra del o la articulista para concluir con lo que quiere sostener: la Seńorita Cecilia Grierson "[...] abre nuevos horizontes a la actividad intelectual de la mujer argentina, nuevo campo en que ejercitar las facultades de su espíritu."

Mientras estas conferencias y artículos fundamentaban la capacidad de la mujer para desempeńarse en cualquier actividad productiva y en cualquier nivel educativo, además del magisterio, en otro artículo sin firma (pero seguramente de un educador o educadora miembro de la ANE), se cuestiona la capacidad de la mujer como maestra. ${ }^{45}$ Pero eso no es todo, si en la conferencia de la maestra mendocina se subraya que la mujer puede emanciparse incorporándose al mundo del trabajo sin descuidar su función de madre de familia, en este artículo se afirma que la mayoría de las maestras no le interesa el magisterio porque no tienen vocación, y que es el casamiento lo único que le interesa. ${ }^{46}$ ¿Qué permiten ver estas disputas verbales reproducidas en las páginas de La Educación? La existencia de un arduo debate entre los mismos miembros de la ANE. No se observa una sola voz de los nuevos educadores sino un coro de voces, y en este caso con posiciones diametralmente diferentes y duramente enfrentadas.

\section{Consideraciones finales}

Los estudios clásicos y los más recientes sobre la historia del normalismo en el siglo XIX, enfocan su mirada en las Memorias de Instrucción Pública, en publicaciones oficiales, y en publicaciones periódicas masivas. Con estas fuentes han producido, sin duda, investigaciones que iluminan muchos aspectos del normalismo argentino. Sin embargo, ha escapado en la mayoría de los investigadores el examen de las discusiones de los nuevos educadores en las múltiples asociaciones creadas en las dos últimas décadas del siglo XIX. Mi investigación, con el objeto de ampliar la mirada que se tenía sobre los educadores y en especial de los normalistas, fija la observación en las publicaciones de estas asociaciones, y en particular de la ANE. De este modo, he advertido que los nuevos educadores (quiénes pretenden ocupar los espacios más importante de decisión en el campo educativo) se instalan en la sociedad civil para dar sus discusiones, y para presentarse como un actor en el campo educativo y, sobre todo, en el campo normalista. Crean asociaciones y desde ellas sientan sus posiciones ante los poderes públicos, y en particular, para disputar las normas de percepción que

coronado hoy sus esfuerzos con el título hermoso de Doctora en Medicina y Cirugía que la Facultad, le discierne, no sin haber pasado antes por todas las pruebas que los Estatutos Universitarios imponen para acreditar la competencia e idoneidad del estudiante. Doctora formada no solo con el estudio inteligente de libros, sino con la observación constante de los organismos en el anfiteatro, en la sala de disección, en las salas de los hospitales, etc., posee a la par de la preparación teórica, la práctica indispensable, absolutamente necesario, para ejercer con acierto la ciencia de los Esculapios e Hipócrates. Es, pues, un médico ilustrado que hará honor a su gremio y bien a la sociedad que actúa [...] Es un trabajo, según opiniones autorizadas, de verdadero mérito por lo prolijo de las observaciones. Unimos nuestra palabra de felicitación a las muchas que se han dedicado ya a la primer doctora argentina y levantamos nuestra humilde voz para celebrar el hecho de importancia trascendental, que abre nuevos horizontes a la actividad intelectual de la mujer argentina, nuevo campo en que ejercitar las facultades de su espíritu." Ibidem.

44 “[...] No carecen de interés los siguientes párrafos de la introducción de su tesis: "Antes de entrar en materia, permítaseme dos palabras: como primer mujer argentina que sube a esta tribuna quiero decir lo que siento; y que ello sirva de aliento a las que vendrán después. Las dificultades que he encontrado en mi carrera han sido menos de las que esperaba; solo palabras de gratitud tengo para mis maestros, mis condiscípulos y amigos; todos, y cada uno han tenido atenciones y delicadezas, que solo un hermano puede prodigar". Ibidem.

45 “Las mujeres como maestras” en: La Educación. Periódico Quincenal, enero 15 y febrero 1 de 1888. Año II, n. 44 y 45 , p. 733.

${ }^{46}$ Ibidem. 
intentan imponer los fundadores de las escuelas normales o los fisiólogos que estudian temas relacionados con la educación y la educación de la mujer. Discutir con los fundadores de las escuelas normales o con fisiólogos implicaba para ellos posicionarse además en el campo normalista y en el campo científico, con el fin de autorizar su palabra sobre temas educativos. Las fuentes seleccionadas permiten advertir, además, de qué manera estos nuevos educadores usan la ciencia para describir la realidad educativa y para legitimar sus acuerdos y sus enfrentamientos con los fundadores del normalismo. Su voluntad normalista impera siempre, (es decir, la defensa de los valores e intereses del normalismo), y los convierte en continuadores de los fundadores de las escuelas normales aunque invocan otro saber y otras prácticas que los lleva a redefinir cuestiones básicas: por ejemplo, el salario que deben cobrar o los cargos que deben ocupar las maestras en sede educativa, o proponer escuelas de artes y oficios mal vistas por los fundadores del normalismo, pero agregando un plus: se plantea la creación de escuelas de artes y oficios para mujeres. Estas fuentes permiten observar también que no se trata, tal como se aprecia en los debates analizados de la ANE, de una asociación que expone un discurso homogéneo, sino que existen discusiones entre sus miembros, no se puede hablar de nuevos educadores como una unidad, sino como un conjunto de voces, tienen puntos sustanciales que los unen y que defienden, y puntos que distancian a unos de otros, conviven, para decirlo de otro modo, los encuentros y los desencuentros visibles en las páginas de las publicaciones de sus asociaciones y en sus prácticas educativas. Esta última consideración resulta significativa no como punto de llegada sino como punto de partida de mi investigación sobre el campo normalista argentino (más claramente de las asociaciones normalistas) de fines del siglo XIX.

\section{Bibliografía}

\section{Publicaciones periódicas.}

- La Escuela Positiva, Revista Mensual, Corrientes, 1895-1896.

- La Educación. Periódico Quincenal, Órgano de la Asociación Nacional de Educación, Buenos Aires, 18861897.

— Revista Pedagógica Argentina, Órgano del Centro Unión Normalista, Buenos Aires, 1889-1891.

\section{Censos.}

- Censo escolar del Distrito IV. Noviembre de 1899. Publicación del Consejo Nacional de -Educación por el Dr. Joaquin V. González, vocal del Consejo, (1900). Félix Lajouane Editor, Buenos Aires.

\section{Memorias Oficiales}

- Memoria presentada al Congreso Nacional de 1881 por el ministro de Justicia e Instrucción Pública Doctor Manuel D. Pizarro, Buenos Aires, Imprenta de la Penitenciaria, 1881.

\section{Libros}

- Alliaud Andrea. (1993): Los maestros y su historia: los origenes del magisterio argentino, Buenos Aires, Centro Editor de América Latina.

—Barrancos Dora, (1990): Anarquismo, educación y costumbres en la Argentina de principios de siglo, Buenos Aires, Contrapunto, 1990;

(1991): Educación, cultura y trabajadores (1890-1930), Buenos Aires, Centro Editor de América Latina, 1991.

(1993): Historia y Género, Buenos Aires, Centro Editor de América Latina. 
- Bertoni, Lilia Ana. (2000): Patriotas, cosmopolitas y nacionalistas. La construcción de la nacionalidad argentina a fines del siglo XIX. Buenos Aires, Fondo de Cultura Económica.

- Biagini, Hugo, (1983): Educación y Progreso. Primer Congreso Pedagógico Interamericano, Buenos Aires, Academia Nacional de Ciencia.

(1983): La generación del ochenta, Plus Ultra, Buenos Aires.

(1985): El movimiento positivista argentino, Buenos Aires, Editorial de Belgrano.

- Billorou, María José. (1997): "Mujeres en la docencia: una herramienta para la construcción del Estado en el interior argentino (1900-1930)", en: Di Liscia, M. Herminia, y Maristany José (Editores), Mujeres y Estado en la Argentina. Educación, Salud y Beneficencia, Buenos Aires, Biblos.

- Cirigliano, Gustavo, (1967): Educación y futuro, Columba, Buenos Aires.

(1969): Educación y politica; el paradojal del sistema de la educación pública argentina, Librería del Colegio, Buenos Aires.

Colmenar Orzares, Carmen. (1983): "Contribución de la escuela normal central de maestros a la educación femenina en el siglo XIX”, en: Historia de la Educación, Tomo 2.

— Chavarría, José Manuel. (1947): La escuela normal y la cultura argentina, El Ateneo, Buenos Aires.

- Di Tella, Torcuato. (1965) "Raíces de la controversia educacional en Argentina", en: Di Tella y otros. Argentina, sociedad de masas, Eudeba, Buenos Aires.

— Dussel Inés. (1997): Curriculum, humanismo y democracia en la enseñanza media (1863-1920). Buenos Aires, edición UBA-Flacso.

- Herrero, Alejandro. (2010): "La emergencia de un actor en el campo educativo argentino: la nueva escuela normalista”, en: Illapa. Revista Latinoamericana de Ciencias Sociales, Lima, Perú, año 3, n. 7, julio, pp. 85-106.

(2011): "La República Posible y sus problemas en Argentina. Normalistas e industriales debaten el plan educativo alberdiano de las dos gestiones presidenciales de Julio Argentino Roca (1880-1886 y 1898 y 1901)", en Secuencia. Revista de Historia y Ciencias Sociales. Publicación cuatrimestral de Investigaciones Dr. José María Luis Mora, N. 80, mayo-agosto. México, pp. 63-84.

- Finocchio, Silvia. (2009): La escuela en la historia argentina, Buenos Aires, Edhasa, pp. 35-62.

- Filippa, Nely. (2004): Combates por las ideas. El positivismo pedagógico en San Juan (1890-1930), San Juan, Fundación Universidad Nacional de San Juan.

- Katzman, Rubén. (1968): "Estratificación educacional en las provincias Argentinas", en: Desarrollo Económico, n. 28.

- Martínez Paz, Fernando. (1997): “Enseñanza primaria, secundaria y universitaria (1862-1914)", en: Nueva Historia de la Nación Argentina. La configuración de la república independiente (1810-1914), t. 6, Buenos Aires, Planeta.

- Lionetti Lucía. (2007): La misión política de la escuela pública. Formar a los ciudadanos de la república (1870-1916), Buenos Aires, Miño y Dávila editores.

Mauro Diego. (2009): "Católicos, educación y política. La enseñanza religiosa entre la curia diocesana y las orientaciones educativas del estado provincial. Santa Fe, 1915-1937”, en: Estudios Sociales, n. 36, Santa Fe, Universidad Nacional del Litoral.

- Montenegro Ana María. (2012): Un lugar llamado escuela pública. Origen y paradoja (Buenos Aires, 15801911), Buenos Aires, Miño y Dávila.

- Morgade, Graciela (compiladora). (1997): Mujeres en la educación. Género y docencia en la Argentina 1870-1930, Buenos Aires, Mińo y Dávila editores. 
- Muzzopappa Héctor. (2012): Los fundamentos alberdianos y normalistas del proyecto de Osvaldo Magnasco, ministro de Instrucción Pública del gobierno de J. A. Roca (1898-1901). Tesis Doctoral, Doctorado de Filosofía, Departamento de Humanidades y Artes, Universidad Nacional de Lanús.

- Perelstein, Berta, (1952): Positivismo y antipositivismo en Argentina, Procyon, Buenos Aires.

— Puiggrós, Adriana, (1991): Historia de la Educación en la Argentina. Tomo II Sociedad civil y Estado en los orígenes del sistema educativo argentino, Buenos Aires, Editorial Galerna.

(2001): Historia de la Educación en la Argentina. Tomo IV La educación en las provincias y territorios nacionales (1885-1945), Buenos Aires, Editorial Galerna.

Ramos J. P. (1910): Historia de la instrucción primaria de la república argentina (1810-1910), Buenos Aires, Pauser.

- Recalde, Héctor. (1997): La salud de los trabajadores en Buenos Aires (1870-1910). A través de las fuentes médicas. Buenos Aires, Grupo Editor Universitario.

Roig, Arturo A. (2006): Los krausistas argentinas. Edición corregida y aumentada, Buenos Aires, Ediciones El Andariego, pp. 86-113.

- Tedesco, Juan Carlos. (1970): Educación y sociedad en Argentina (1880-1900), Editorial Pannedile, Buenos Aires.

(1973a): "Educación e ideología en Argentina. Notas para una investigación”, en: Los libros. Para una crítica de la cultura politica, Buenos Aires, pp. 4-12.

(1973b): "El positivismo pedagógico argentino", en: Revista Paraguaya de Sociología, Asunción, n. 26, pp. $37-48$.

(1980): "La educación argentina entre 1880-1930", en: Primera Historia Integral, Buenos Aires, Centro Editor de América Latina.

(1985): "La instancia educativa", en: Bigiani, H. El movimiento positivista argentino, Buenos Aires, Editorial de Belgrano, pp. 333-361.

(2005): Educación popular hoy. Ideas para superar la crisis, Buenos Aires, Capital Intelectual, pp. 9-26.

— Terán, Oscar. (1986), En busca de la ideología Argentina, Buenos Aires, Catálogos.

(1987): Positivismo y nación en Argentina, Buenos Aires, Puntosur.

(2000): Vida intelectual en el Buenos Aires fin-de-siglo (1880-1910). Derivas de la "cultura cientifica", Buenos Aires, Fondo de Cultura Económica.

(2008): Historia de las ideas en la Argentina. Diez lecciones iniciales, 1810-1980, Buenos Aires, Siglo XXI.

- Sarmiento, D. F., "Nota del Superintendente General de la Educación sobre la creación de una Escuela de Artes y Oficios", en: Memoria presentada al Congreso Nacional de 1881 por el ministro de Justicia e Instrucción Pública Doctor Manuel D. Pizarro, Buenos Aires, Imprenta de la Penitenciaria, 1881, pp. 80 y ss.

"Aptitudes industriales", en: Obras completas de Domingo Faustino Sarmiento. Discursos populares. Segundo volumen, Tomo XXII, Buenos Aires, Universidad Nacional de La Matanza, 2001, pp. 225-238.

- Szir Sandra. (2007): Infancia y cultura visual. Los periódicos ilustrados para niños (1880-1910), Buenos Aires, Miño y Dávila.

Soler, R. (1959): El positivismo argentino, Panamá, Imprenta Nacional.

—Zimmermann, Eduardo. (1995): Los liberales reformistas. La cuestión social en la Argentina, 1890-1916, Buenos Aires, Editorial Sudamericana-Universidad de San Andrés. 